\title{
Chassis 2025 - strategic key competence or commodity?
}

Thomas Müller, M. C. Paefgen, AUDI AG

This manuscript is not available according to publishing restriction. Thank you for your understanding. 